\title{
Fatores etiológicos e prevalência de lesões bucofaciais em surfistas de Fortaleza
}

\author{
Jivago Barreto França Cordeiro ${ }^{a, *}$, Luceana Barreira Forte ${ }^{b}$, Jiovanne Rabelo Neria,b ${ }^{a}$, Saulo Ellery Santos ${ }^{b}$, Fábio \\ de Almeida Gomes ${ }^{b}$, Danilo Lopes Ferreira Limaab
}

\section{Palavras Chave: Odontologia; \\ Esporte; \\ Traumatismo em desportistas; \\ Boca.}

\begin{abstract}
RESUMO
O estudo investigou fatores etiológicos e prevalência de lesões bucofaciais em surfistas profissionais e amadores em Fortaleza, Ceará. Aplicou-se um questionário em 150 surfistas homens e usaramse testes estatísticos (qui-quadrado e teste $t$ de Student) com nível de significância $p<0,05$. Em 56\% encontrou-se alguma lesão bucofacial. Queimaduras de lábio e de face, laceração de mucosa e fratura dentária foram as mais prevalentes. Comparando surfistas competidores com não competidores houve significância entre ocorrência de lesões com fundo de rocha $(p=0,032)$ e com prancha $(p=0,003)$ e maior presença de lesões entre os competidores $(p=0,003)$. Concluise que fraturas dentárias e queimaduras facial e labial são prevalentes em surfistas e que há mais chances de ocorrência de lesões quanto maiores forem a idade e o tempo de prática.
\end{abstract}

\begin{abstract}
The study investigated etiological factors and the prevalence of orofacial lesions in surfers professionals and amateurs in Fortaleza, Brazil. A questionnaire was applied to 150 male surfers and statistical tests (Chi-square and Student $t$ ) were used with significance level $p<0.05$. In $56 \%$, there was some orofacial lesion. Lip and face burns, mucosal laceration and dental fracture were the most prevalent. Comparing competitive surfers with non-competitors there was significance between the occurrence of rock bottom injuries $(p=0.032)$ and surfboard $(p=0.003)$ and greater presence of injuries among competitors $(p=0.003)$. It is concluded that dental fractures and facial and lip burns are prevalent in surfers and that there is a greater chance of occurrence of injuries the greater the age and the time of practice of them.
\end{abstract}

\section{RESUMEN}

El estudio investigó factores etiológicos y prevalencia de lesiones bucofaciales en surfistas profesionales y aficionados en Fortaleza, Ceará. Se aplicó un cuestionario en 150 surfistas de sexo masculino y se utilizaron pruebas estadísticas (chi cuadrada y prueba de la t de Student) con un nivel de relevancia estadística de $p<0,05$. En el $56 \%$ se encontró alguna lesión bucofacial. Las más frecuentes fueron quemaduras del labio y de la cara, laceración de mucosa y fractura dental. Al comparar a surfistas competidores con otros no competidores hubo relevancia entre lesiones producidas por el fondo $(p=0,032)$ y por la tabla $(p=0,003)$, y mayor existencia de lesiones entre competidores $(p=0,003)$. Se concluye que las fracturas dentales y las quemaduras facial y labial son frecuentes en surfistas y hay más probabilidad de que se produzcan lesiones cuanto mayor es la edad y el tiempo de práctica.

\footnotetext{
a Universidade de Fortaleza (Unifor), Curso de Odontologia, Fortaleza, CE, Brasil

${ }^{\text {b } C e n t r o ~ U n i v e r s i t a ́ r i o ~ C h r i s t u s ~(U n i c h r i s t u s), ~ C u r s o ~ d e ~ O d o n t o l o g i a, ~ F o r t a l e z a, ~ C E, ~ B r a s i l ~}$
}

* Correspondence author:

Jivago Barreto França Cordeiro

E-mail: jivagodonto@gmail.com

Recebido em 7 de janeiro de 2018; aceito em 15 de março de 2018. 


\section{INTRODUÇÃO}

A conscientização em relação à prevenção de doenças e a necessidade de melhorar a qualidade de vida proporcionaram um aumento significativo no número de pessoas que praticam atividades físicas em todo o mundo (Sahlin e Lexell, 2015, Costa et al., 2018). Nesse contexto, o surfe destacou-se por ser um esporte aquático praticado diretamente em contato com a natureza, sem necessitar de instalações físicas convencionais, permite que seus praticantes experimentem o alívio das tensões diárias, a sensação de liberdade e o estabelecimento de relações sociais gratificantes (Romariz et al., 2011). No Brasil, mais especificamente, a presença de uma longa faixa litorânea, com mais de $4.000 \mathrm{~km}$ de extensão, e as boas condições climáticas favoreceram o crescimento do surfe como uma opção saudável para a obtenção de um perfeito equilíbrio entre o corpo e a mente (Steinman et al., 2000).

A prática do surfe, tanto em nível amador como profissional, exige reflexos rápidos e capacidade física apurada para que os praticantes desenvolvam manobras perfeitas (Vaghettietal.,2007, Farleyetal.,2017). Adicionalmente, o desempenho dos atletas é influenciado também pelas condições do ambiente oceânico, como a ação dos ventos, as diferentes correntes marítimas, o tipo de fundo do oceano, a ação da gravidade da lua sobre as marés, o tamanho das ondas e a temperatura ambiente (Moraes et al., 2013, Minghelli et al., 2017). Portanto, a combinação de todas essas variáveis representa um perigo à saúde do atleta, pois aumenta exponencialmente o risco de ocorrência de traumas e lesões (Steinman et al., 2000; Moraes et al., 2013; Base et al., 2007, Minghelli et al., 2017).

A cabeça e a face são os locais mais prevalentes de lesões entre os surfistas (Furness et al., 2015). A maioria das lesões bucofaciais ocorridas em atletas, em geral, é localizada nos lábios superiores e na maxila e os traumatismos dentários ocorrem, principalmente, nos incisivos centrais superiores (Emerich e Kaczmarek, 2010; Welch et al., 2010).

A atuação do cirurgião-dentista juntamente com uma equipe multidisciplinar pode melhorar a atuação do atleta em suas atividades, visto que é cada vez mais evidente a estreita relação entre a odontologia e as práticas esportivas (Lima, 2012, Goswami et al., 2017). Consequentemente, o Conselho Federal de Odontologia criou uma nova especialidade denominada de odontologia do esporte, com a função de estudar, prevenir e tratar as lesões que afetam o sistema estomatognático, bem como manter a saúde bucal de atletas (CFO, 2015). Embora o surfe seja um esporte muito praticado no mundo, a produção científica sobre essa modalidade é escassa, no Brasil, e pouco se sabe a respeito das principais lesões bucofaciais que acometem os surfistas. Assim, o objetivo do presente estudo foi investigar os fatores etiológicos e a prevalência de lesões bucofaciais em surfistas profissionais e amadores em Fortaleza.

\section{MÉTODO}

O presente estudo, com delineamento observacional transversal, foi feito com 150 praticantes de surfe do sexo masculino. Foram incluídos surfistas amadores e profissionais, independentemente da idade, e que praticavam o esporte havia, pelo menos, um ano. Foram excluídos aqueles que praticam o esporte menos de duas vezes por semana. Os sujeitos foram informados dos procedimentos experimentais e assinaram o Termo de Consentimento Livre e Esclarecido, aprovado pelo Comitê de Ética da Universidade de Fortaleza sob parecer $n^{\circ} 142 / 11$.

Para a coleta de dados foi usado um questionário composto por perguntas fechadas, dicotômicas e de múltipla escolha. As variáveis avaliadas foram: idade; frequência semanal de prática; tempo de prática; participação em competições como amador ou profissional (sim; não); uso de protetor bucal para prevenção de lesões dentoalveolares (sim; não); uso de protetor solar para a face (sim; não); uso de protetor solar para os lábios (sim; não); ocorrência de lesão associada à prática esportiva (sim; não); tipo de lesão (fratura dentária; fratura de ossos da face; laceração de mucosa; queimadura do lábio; queimadura da face; avulsão dentária); forma de ocorrência da lesão (prancha; fundo de rocha; fundo de coral; fundo de areia; animal marinho; choque com surfista; choque com banhista; raios solares). Verificaram-se também aqueles que tiveram mais um tipo de lesão e mais de uma forma de ocorrência. Os dados foram coletados no ambiente da própria prática do esporte, a praia, ocasião na qual os sujeitos foram devidamente informados sobre o estudo, seus objetivos e benefícios.

Os dados foram tabulados e os cálculos estatísticos foram feitos com o Programa Statistical Package for the Social Science (SPSS) na versão 22.0 (SPSS Inc., Chicago, Estados Unidos). A normalidade da distribuição de cada variável foi avaliada por meio do teste de Kolmogorov-Smirnov. O teste qui-quadrado com nível de significância $p<0,05$ foi usado para associações das variáveis categóricas. Para a comparação da idade, tempo de prática e frequência semanal de treino entre as variáveis nominais foi usado o teste $t$ para amostras independentes. 


\section{RESULTADOS}

Entre os 150 surfistas investigados, as idades variaram entre 12 e 56 anos, com média de 28,3 $\pm 8,1$ anos. Quanto ao tempo de prática, variou entre um e 36 anos, com média de 10,6 \pm 7 anos, e a frequência semanal de treino média foi de $3,4 \pm 1,5$ dias. Participavam de competições esportivas 66 (44\%) surfistas, enquanto 84 (56\%) praticavam o esporte somente por lazer. Entre os que participavam de campeonatos, tanto a média de idade $(30,6 \pm 9$ anos) quanto o tempo de prática $(13,6 \pm 7,8$ anos) foram maiores do que os que praticavam o surfe de forma recreacional $(26,5 \pm 6,9$ e $8,3 \pm 5,2$, respectivamente).

Nenhum surfista usava protetor bucal, somente 36 (24\%) faziam uso de protetorfacial e 30 (20\%) de protetor labial. Reportaram algum tipo de lesão bucofacial 84 (56\%) investigados, 42 (28\%) sofreram mais de um tipo de lesão (Tabela 1). Quando comparado o grupo de surfistas que fazia parte de competições como amador ou profissional com o que surfava somente por lazer verificou-se uma maior presença de lesões $(p=0,003)$ entre os que competiam, as mais significativas foram as lacerações de mucosa $(p=0,001)$, fraturas dentárias $(p=0,023)$ e mais de um tipo de lesão $(p=0,043)$.

Quando verificado como ocorreu a lesão, 50 (33,3\%) indivíduos afirmaram ter sofrido lesões em decorrência de impacto com a prancha, $38(25,3 \%)$ através de raios solares e 21 (14\%) surfistas tiveram mais um tipo de ocorrência. Ao comparar os grupos de competidores e não competidores, a ocorrência de lesões com fundo de rocha $(p=0,032)$ e com prancha $(p=0,003)$ foi significativa (Figura 1 ).

Quando investigada a relação das lesões com a idade, tempo de prática e frequência semanal de treino observou-se que indivíduos mais velhos têm uma maior presença de lesões $(p=0,024)$, notadamente as fraturas dentárias (idade $p=0,001$ ) e as fraturas de osso da face $(p=0,05)$. Não foi observada significância entre a frequência semanal de treino e qualquer tipo de lesão. Já o tempo maior de prática estava relacionado às fraturas dentárias $(p=0,007)$.
Forma de ocorrência da lesão

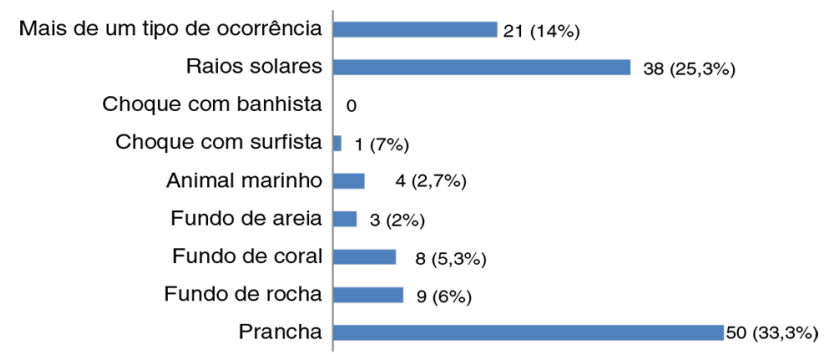

Figura 1.

No tocante à forma de ocorrência dos acidentes, os que aconteceram em fundo de rocha relacionaramse com a idade $(p=0,001)$ e com o tempo de prática $(p=0,001)$. O fundo de coral teve significância $p=0,001$, com ambos, idade e tempo de prática, assim como mais de uma forma de ocorrência, com $p=0,001$ e $p=0,01$ respectivamente. Lesões ocorridas pelo contato com animais marinhos foram significantes entre aqueles com maior tempo de prática $(p=0,01)$. Assim como nas lesões, a frequência semanal de prática não foi significativa quando comparada à forma de ocorrência das lesões.

\section{DISCUSSÃO}

A prática do surfe tem se popularizado muito nos últimos anos. Estima-se que haja 17 milhões de surfistas em todo o mundo, enquanto no Brasil o número total de praticantes do esporte é de aproximadamente 2,7 milhões (Base et al., 2007). O surfe é praticado por atletas de todas as idades, contudo a média de idade dos surfistas varia entre 20 e 30 anos (Steinman et al., 2000; Moraes et al., 2013; Base et al., 2007). A maior parte dos praticantes desse esporte o faz como uma forma de lazer, ou seja, sem a responsabilidade de treinos regulares ou pressões por competição (Steinman et al., 2000; Moraes et al., 2013). Esses dados corroboram os achados do presente estudo, uma vez que a média de idade foi de $26,5 \pm 6,9$ anos e $56 \%$ eram surfistas recreacionais.

Por outro lado, durante uma competição, os surfistas são julgados pela sua capacidade de enfrentar

Tabela 1. Tipos de lesões bucofaciais ocorridas em surfistas.

\begin{tabular}{lcccc}
\hline Tipo de lesão & Sim & Não & Amadores & Profissionais \\
\hline Fratura dentária $^{a}$ & $30(20 \%)$ & $120(80 \%)$ & $7(8,3 \%)$ & $11(16,7 \%)$ \\
Fratura de ossos da face $^{\text {Laceração de mucosa }}$ & $3(2 \%)$ & $147(98 \%)$ & $1(1,2 \%)$ & $0(0 \%)$ \\
Queimadura do lábio & $33(22 \%)$ & $117(78 \%)$ & $4(4,8 \%)$ & $10(15,2 \%)$ \\
Queimadura da face & $38(25,3 \%)$ & $112(74,7 \%)$ & $2(2,4 \%)$ & $1(1,5 \%)$ \\
Avulsão dentária & $33(22 \%)$ & $117(78 \%)$ & $1(1,2 \%)$ & $0(0 \%)$ \\
Mais de um tipo de lesão $^{a}$ & $8(5,3 \%)$ & $142(94,7 \%)$ & $1(1,2 \%)$ & $1(1,5 \%)$ \\
\hline
\end{tabular}

${ }^{a} p<0,05$. 
as mudanças na formação das ondas e conseguir executar manobras com perfeição nas diversas sessões (Lowdon e Pateman, 1980). Nessa situação, muitos fatores podem interferir diretamente no desempenho do atleta, tais como a frequência de treinamento, o condicionamento físico e o tempo de experiência no esporte (Vaghetti et al., 2007). Assim, é perfeitamente plausível que entre os surfistas competidores, profissionais e amadores o tempo de prática $(13,6 \pm 7,8$ anos) tenha sido maior do que o dos surfistas recreacionais ( $8,3 \pm 5,2$ anos).

Como o surfe é um esporte praticado ao ar livre e em cidades de clima quente, os cuidados com a pele dos atletas deveriam ser uma rotina, a partir do uso de agentes protetores contra os raios ultravioletas do sol (Dobbinson et al., 2008; Purim e Leite, 2010). Entretanto, o presente estudo observou que apenas uma pequena quantidade dos surfistas entrevistados fazia uso de protetores facial $(24 \%)$ e labial $(20 \%)$, o que corroborou os achados de Meir et al. (2012). A ocorrência de lesões cutâneas em pessoas que se expõem ao sol pode estar associada a fatores comportamentais e ambientais (Dobbinson et al., 2008; Jadotte e Schwartz, 2012; Olsen et al., 2015; Heckman et al., 2016). Provavelmente, o hábito de não usar proteção contra os raios ultravioletas do sol associado às questões ambientais locais de Fortaleza, uma cidade próxima à linha do Equador, com alta temperatura média $\left(26,7^{\circ} \mathrm{C}\right)$ e com taxa de insolação anual de $2.843,4$ horas (Instituto Nacional de Meteorologia, 2015), pode ter ocasionado as queimaduras faciais e labiais entre os surfistas.

Durante a prática do surfe, o organismo pode sofrer agressões de variados modos, origens, frequências e intensidade (Steinman et al., 2000). Em um estudo conduzido com 1.348 surfistas, 37\% tiveram lesões na região de cabeça e face (Nathanson et al., 2007). Entre as lesões bucofaciais, as mais prevalentes são as lesões cortocontundentes em lábios e as fraturas dentárias (Emerich e Kaczmarek, 2010; Welch et al., 2010), o que corrobora os achados no presente estudo (Tabela 1). A prancha, por estar sempre em contato com o surfista e ligada a ele pelo leasch, tende a ser um instrumento com maior possibilidade de causar lesões (Figura 1). Esses dados concordam com os achados por Nathanson et al. (2007), os quais verificaram que $55 \%$ das lesões em face e cabeça deram-se por contato do surfista com a própria prancha e $12 \%$ pelo contato com a prancha de outro surfista.

Por outro lado, as condições ambientais do mar onde se pratica o surfe também implicam aumento no risco de lesões (Moraes et al., 2013). A presença de fundo de coral aumentou em 2,4 vezes o risco de lesões em surfistas durante as competições
(Nathanson et al., 2007). Embora o fundo do mar do Ceará seja caracterizado pela presença de areia ou coral, no presente estudo foi observado que houve uma relação significante entre os acidentes com surfistas mais velhos e com maior tempo de prática em fundo de rocha (Figura. 1). É possível que esse fato seja decorrente da oportunidade que muitos surfistas têm de surfar em outros estados e até em outros países que têm o fundo do mar de rocha.

Uma opção viável para evitar a ocorrência de fraturas dentárias seria o uso de protetores bucais (Magunacelaya e Glendor, 2011; Newsome et al., 2001; Tiwari et al., 2014). Contudo, o uso de protetores bucais ainda está bem restrito aos esportes de contato, principalmente as lutas (Newsome et al., 2001; Tiwari et al., 2014). Atletas e praticantes de determinados esportes, como o surfe, no qual não existe um histórico de uso de protetores bucais, continuam relutantes em usar o dispositivo (Tabela 1). Dessa forma, seria interessante uma campanha de conscientização para que todas as medidas preventivas de lesões bucofaciais fossem implantadas para os atletas do surfe e de outras modalidades que usam pranchas.

Pode-se concluir que fraturas dentárias e queimaduras facial e labial são prevalentes em surfistas. Quanto maior a idade e o tempo de prática, mais chances de ocorrência de lesões, principalmente ocasionadas pela prancha. Medidas educativas e preventivas devem ser implantadas, visto que o surfe é um dos esportes mais praticados no Brasil e no mundo.

\section{FINANCIAMENTO}

O presente trabalho não contou com apoio financeiro de qualquer natureza para sua elaboração.

\section{CONFLITO DE INTERESSES}

Os autores declaram não haver conflitos de interesse.

\section{REFERÊNCIAS}

Base LH, Alves MAF, Martins EO, et al. Lesões em surfistas profissionais. Rev Bras Med Esporte 2007;13:251-3.

Conselho Federal De Odontologia (CFO), 2015. [Acesso em

16 nov.2015.] Disponível em <http://www.normaslegais. com. br/legislacao/Resolucao-cfo-160-2015.htm>.

Costa EC, de Sá JCF, Stepto NK, et al. Aerobic Training Improves Quality of Life in Women with Polycystic Ovary Syndrome. Med Sci Sports Exerc 2018:13.

Dobbinson S, Wakefield M, Hill D, et al. Prevalence and determinants of Australian adolescents' and adults' weekend sun protection and sunburn, summer 2003-2004.J Am Acad Dermatol 2008;59:602-14.

Emerich K, Kaczmarek J. First aid for dental trauma caused by sports activities: state of knowledge, treatment and prevention. Sports Med 2010;40:361-6. 
Farley OR, Abbiss CR, Sheppard JM. Performance Analysis of Surfing: A Review. J Strength Cond Res 2017;31:260-71.

Furness J, Hing W, Walsh J, Abbott A, et al. Acute injuries in recreational and competitive surfers: incidence, severity, location, type, and mechanism. Am J Sports Med 2015;43:1246-54.

Goswami M, Kumar P, Bhushan U. Evaluation of Knowledge Awareness, and Occurrence of Dental Injuries in Participant Children during Sports in New Delhi: A Pilot Study. Int J Clin Pediatr Dent 2017;10:373-8.

Heckman CJ, Darlow SD, Ritterband LM, et al. Efficacy of an Intervention to Alter Skin Cancer Risk Behaviors in Young Adults. Am J Prev Med 2016;51:1-11.

Instituto Nacional de Meteorologia. [Acesso em: 5 dez.2015.] Disponível em: <http://www.inmet.gov.br/portal/index. php?r=clima/normaisClimatologicas>.

Jadotte YT, Schwartz RA. Solar cheilosis: an ominous precursor: part I Diagnostic insights. J Am Acad Dermatol 2012;66:173-84.

Lima DLF. Odontologia Esportiva: o cirurgião-dentista no cuidado do esportista. São Paulo: Editora Santos; 2012.

Lowdon BJ, Pateman NA. Physiological parameters of international surfers. Aust J Sports Med 1980;12:34-9.

Magunacelaya MB, Glendor U. Surfing for mouth guards: assessing quality of online information. Dent Traumatol 2011;27:334-43.

Meir RA, Zhou S, Gilleard WL, et al. An investigation of surf injury prevalence in Australian surfers: A self-reported retrospective analysis. NZJSM 2012;39:52-8.

Minghelli B, Nunes C, Oliveira R. Injuries in recreational and competitive surfers: a nationwide study in Portugal. J Sports Med Phys Fitness 2017;24:9.

Moraes GC, Guimaraes ATB, Gomes ARS. Análise da prevalência de lesões em surfistas do litoral paranaense. Acta Ortop Bras 2013;21:213-8.
Nathanson A, Bird S, Dao L, Tam-Sing K. Competitive surfing injuries: a prospective study of surfing-related injuries among contest surfers. Am J Sports Med 2007;35:113-7.

Newsome PR, Tran DC, Cooke MS. The role of the mouthguard in the prevention of sports-related dental injuries: a review. Int J Paediatr Dent 2001;11:396-404.

Olsen CM, Thompson BS, Green AC, et al. Protection and Skin Examination Practices in a Setting of High Ambient Solar Radiation: A Population-Based Cohort Study. JAMA Dermatol 2015;151:982-90.

Purim KSM, Leite N. Fotoproteção e exercício físico. Rev Bras Med Esporte 2010;16:224-9.

Romariz JK, Guimarães ACA, Marinho A. Qualidade de vida relacionada à prática de atividade física de surfistas. Motric 2011;17:477-85.

Sahlin KB, Lexell J. Impact of Organized Sports on Activity Participation, and Quality of Life in People With Neurologic Disabilities. PM R 2015;7:1081-8.

Steinman J, Vasconcellos EH, Ramos RM, et al. Epidemiologia dos acidentes no surfe no Brasil. Rev Bras Med Esporte 2000;6:9-15.

Tiwari V, Saxena V, Tiwari U, et al. Dental trauma and mouthguard awareness and use among contact and noncontact athletes in central India. J Oral Sci 2014;56:239-43.

Vaghetti CAO, Roesler $\mathrm{H}$, Andrade A. Tempo de reação simples auditivo e visual em surfistas com diferentes níveis de habilidade: comparação entre atletas profissionais, amadores e praticantes. Rev Bras Med Esporte 2007;13:81-5.

Welch CL, Thomson WM, Kennedy R. ACC claims for sportsrelated dental trauma from 1999 to 2008: a retrospective analysis. N Z Dent J 2010;106:137-42. 